\title{
Alteration of mitochondrial biogenesis promotes disease progression in multiple myeloma
}

\author{
Xin Zhan ${ }^{1, *}$, Wenjie Yu${ }^{2, *}$, Reinaldo Franqui-Machin ${ }^{1,3}$, Melissa L. Bates ${ }^{4}$, Kalyan \\ Nadiminti ${ }^{1}$, Huojun Cao ${ }^{5}$, Brad A. Amendt ${ }^{2,5}$, Yogesh Jethava ${ }^{1}$, Ivana Frech ${ }^{1}$, \\ Fenghuang Zhan ${ }^{1}$ and Guido Tricot $^{1}$ \\ ${ }^{1}$ Division of Hematology, Oncology, and Blood and Marrow Transplantation, Department of Internal Medicine, University of \\ Iowa, Iowa City, IA, USA \\ ${ }^{2}$ Department of Anatomy and Cell Biology University of Iowa, Iowa City, IA, USA \\ ${ }^{3}$ Molecular \& Cellular Biology Program, University of Iowa, Iowa City, IA, USA \\ ${ }^{4}$ Department of Health and Human Physiology, University of Iowa, Iowa City, IA, USA \\ ${ }^{5}$ Department of Endodontics University of Iowa, Iowa City, IA, USA \\ *These authors have contributed equally to this work \\ Correspondence to: Fenghuang Zhan, email: fenghuang-zhan@viowa.edu \\ Keywords: multiple myeloma; mitochondria biogenesis; iron; disease progression \\ Received: August 24, $2017 \quad$ Accepted: October 13,2017 Published: November 27, 2017 \\ Copyright: Zhan et al. This is an open-access article distributed under the terms of the Creative Commons Attribution License 3.0 \\ (CC BY 3.0), which permits unrestricted use, distribution, and reproduction in any medium, provided the original author and source \\ are credited.
}

\section{ABSTRACT}

Many cancers, including multiple myeloma (MM), retain more cytosolic iron to promote tumor cell growth and drug resistance. Higher cytosolic iron promotes oxidative damage due to its interaction with reactive oxygen species generated by mitochondria. The variation of mitochondrial biogenesis in different stages of MM disease was evaluated using gene expression profiles in a large clinical dataset. Sixteen of $18 \mathrm{mitochondrial} \mathrm{biogenesis} \mathrm{related} \mathrm{gene} \mathrm{sets,} \mathrm{including} \mathrm{mitochondrial}$ biogenesis signature and oxidative phosphorylation, were increased in myeloma cells compared with normal plasma cells and high expression was associated with an inferior patient outcome. Relapsed and drug resistant myeloma samples had higher expression of mitochondrial biogenesis signatures than newly diagnosed patient samples. The expression of mitochondrial biogenesis genes was regulated by the cellular iron content, which showed a synergistic effect in patient outcome in MM. Pharmacological ascorbic acid induced myeloma cell death by inhibition of mitochondria oxidative phosphorylation in an in vivo model. Here, we identify that dysregulated mitochondrial biogenesis and iron homeostasis play a major role in myeloma progression and patient outcome and that pharmacological ascorbic acid, through cellular iron content and mitochondrial oxidative species, should be considered as a novel treatment in myeloma including drug-resistant and relapsed patients.

\section{INTRODUCTION}

Multiple myeloma (MM) is a difficult to cure plasma cell tumor. It is the second most common blood malignancy. High-dose chemotherapy approaches, including proteasome inhibitors, immunomodulatory drugs (IMiDs), alkylating agents, and glucocorticoids with tandem auto-transplants in recently diagnosed MM patients have led to complete remissions (CRs) in the large majority of newly diagnosed patients with MM. However, many patients who achieve CR subsequently relapse. We recently showed that pharmacological ascorbic acid 
(PAA) as a pro-oxidant agent selectively kills MM cells by generating ascorbyl- and $\mathrm{H}_{2} \mathrm{O}_{2}$ radicals in cancer cells [1] PAA-induced cell death depends on cellular iron content and cleavage of apoptosis-inducing factor 1 (AIF1), a mitochondrial biogenesis related gene [1]. Furthermore, increasing intracellular steady-state pro-oxidant levels in stem-like and mature MM cells using a mitochondrialtargeting agent decyl-triphenylphosphonium (10-TPP) promotes cancer cell death [2]. Though many pre-clinical studies for targeting mitochondria have been reported in MM [3, 4], it remains unclear whether mitochondrial biogenesis is beneficial or detrimental for tumor cells. A better understanding of the genetic makeup of mitochondria in MM cells is required if we want to use mitochondria as specific targets.

In cancer cells, mitochondria generate ATP through both oxidative phosphorylation (OXPHOS) and aerobic glycolysis (the Warburg effect) for cell proliferation [5]. Both mitochondrial biogenesis and mitophagy (mitochondrial autophagy) control mitochondrial content and metabolism $[6,7]$. In cancer cells, mitochondrial biogenesis is generally positively regulated by oncogenes and/or negatively by tumor suppressor genes, such as c-Myc and p53 respectively [8, 9]. Mitochondria are organelles where heme and Fe-S clusters are synthesized [10]. Mitochondria are also the major source of reactive oxygen species (ROS), which may induce oxidative damage to DNA, lipids and proteins [8]. Iron acts as an oxidant or reductant by accepting or donating single electrons in a variety of biochemical reactions. Many cancers, including MM, have altered iron metabolism resulting in increased intracellular iron content that facilitates tumor cell growth and drug resistance. Specifically, Ferroportin 1 (FPN1), the only iron exporter in mammalian cells, is decreased in MM tumor cells leading to an increase in the labile iron pool [11]. However, the correlation between these two important pathways that are independently linked to cancer pathogenesis: mitochondrial biogenesis and iron metabolism, has not been investigated in MM disease.

As we described above, the intracellular iron content and mitochondrial biogenesis are important parameters to monitor disease progression and to design novel targeted therapy [12]. Using gene expression profiles (GEP) in a large clinical dataset, we generated seven molecular genetic subgroups and a 70-gene risk model $[13,14]$. These models can predict the genetic changes and prognosis in primary MM patients. Here, we evaluate correlate variations in mitochondrial biogenesis to sensitivity to chemotherapy. We also explore whether dysregulated iron metabolism influences mitochondrial metabolism in MM disease progression. Our data support that mitochondrial biogenesis is increased in drug resistant and relapsed MM cells and PAA can overcome drug resistance via inhibition of mitochondrial oxidative phosphorylation.

\section{RESULTS}

\section{Mitochondrial biogenesis signature is increased in newly diagnosed myeloma patients and associated with poor outcome}

Mitochondrial biogenesis (MitoBio) contains 18 genes from the following categories [12]: 1) mitochondrial membrane integrity (PHB1 and $P H B 2) ; 2$ ) mitochondrial transcription factors (TFAM, TFB1M, and TFB2M); 3) mtDNA replication factors [POLGA, POLGB, and Twinkle (C10orf2)]; 4) co-activators of MitoBio [PPARGC1A (PGC1A), PPARGC1B (PGC1B), and PPRC1]; 5) nuclear respiratory factors $(N R F 1$, and $N F E 2 L 2), 6)$ mitochondrial fission mediators [DRPI (DNMIL) and FIS1], 7) mitochondrial fusion mediators (MFN1 and MFN2); and 9) other regulators of MitoBio (ESRRA). We collected plasma cells from healthy donors, tumor cells from Monoclonal Gammopathy of Undetermined Significance (MGUS) and newly diagnosed MM patients using CD138 antibody magnetic beads as previously described, and performed microarrays on Affymetrix U133Plus2 platform [15]. By comparing gene expression profiles of these MitoBio genes from 22 normal plasma cells (NPC) samples and 351 newly diagnosed MM samples in the Total Therapy 2 (TT2) clinical trial, 12 of the above described 18 genes are significantly dysregulated. MFN2, Twinkle, POLGA, TFB2M, FIS1, TFAM, PPRC1, PHB2, NFE2L2, and $P O L G 2\left(0.012>p>1 \times 10^{-10}\right.$ (ordered from the largest to smallest $p$ values $)$ are upregulated and TFB1M $(\mathrm{p}=0.014)$ and NRF1 $(\mathrm{p}=0.049)$ are downregulated (Figure 1A).

To determine whether the elements of the MitoBio profile are linked to patient outcome, Kaplan-Meier survival analysis was performed on the Total Therapy 2 (TT2) and TT3 cohorts, which included gene expression profiles (GEP) from 559 newly diagnosed myeloma patients. The correlation between GEP and survival was determined by the $\mathrm{p}$ value and hazard ratio (HR) at the best expression signal cut-off using the SurvExpress website. High expression of PHB1, PHB2, TFAM, POLG2, TWNK, $P G C 1 A, C R M P 1$, and $M F N 1$ genes were associated with inferior survivals in unadjusted log rank tests (Figure 1B).

A supervised hierarchical cluster was performed on 22 NPC, 44 MGUS and 351 MM samples using the 18 MitoBio genes. As shown in the Figure 2A, a subgroup of MM samples showed a relative high MitoBio signature. We further analyzed the correlation of MitoBio signature with additional 17 mitochondrial gene datasets using the "KEGG Glycolysis and Gluconeogenesis" and "KEGG Oxidative Phosphorylation" as mitochondrial functional controls. A single-sample gene set enrichment analysis (ssGSEA) was performed to calculate the enrichment score for each gene dataset in the 22 NPC, 44 MGUS and 351 MM GEPs. As shown in the Figure 2B, the MitoBio signature was clustered with other 17 mitochondrial gene datasets. Sixteen of 18 mitochondrial gene datasets were 
significantly upregulated in MM cells compared to plasma cells derived from NPC and MGUS, only Glycolysis Gluconeogenesis and Mitochondrial Fatty Acid Beta Oxidation did not show difference between these groups $(*$ in Figure $2 \mathrm{~B}, \mathrm{p}>0.05)$. Interestingly, the oxidative phosphorylation pathway (OXPHOS pathway) was highly correlated with the MitoBio signature $(\mathrm{p}<0.0001)$; whereas the glycolysis gluconeogenesis was not, suggesting that MitoBio may contribute to OXPHOS pathway, but not to glycolysis, for generation of ATP in MM cells.

We calculated a score for each sample using the 18 mitochondrial gene datasets. A correlation between the mitochondrial-score derived from the 18 mitochondrial gene sets with patient outcome was analyzed in the 351 TT2 newly diagnosed MM samples and 264 APEX relapsed MM samples. The group with the higher mitochondrial-score had a significantly inferior event-free survival (EFS) (Figure 2C, $p=0.005$ ) and overall survival (OS) (Figure 2C, $p=0.007$ ) in the TT2 cohort and in the APEX cohort (Figure $2 \mathrm{C}, p<0.001$ ). The correlation between clinical parameters and MitoBio subgroups was also analyzed in the TT2 patients by a univariate analysis (Figure 3A). Patients with a high mitochondrial-score had higher levels of $\beta 2$-microglobulin $(p=0.001)$, lower levels of hemoglobin $(\mathrm{p}=0.035)$, higher percentage of MM cells in bone marrow aspirate $(p=0.007)$, more lytic bone lesions identified by magnetic resonance imaging (MRI; $\mathrm{p}=0.008)$, higher lactate dehydrogenase (LDH; $\mathrm{p}$ $=0.04)$, higher creatinine $(\mathrm{p}=0.006)$, higher frequency of chromosomal metaphase cytogenetics $(\mathrm{p}<0.001)$ with increased hyperdiploid $(\mathrm{p}=0.023)$ and hypodiploid $(\mathrm{p}$ $=0.014)$. The high MitoBio cohort predominated in the high-risk MM group defined by the 70-gene model ( $\mathrm{p}<$ 0.001 ).

\section{Expression of mitochondrial biogenesis genes is higher in drug resistant and relapsed patients}

To investigate whether increased MitoBio signature is increased at relapse, 51 paired GEPs collected at diagnosis and again at relapse were compared using the ssGSEA analysis. The enrichment scores of 7 of 18 mitochondrial data sets are significantly increased in relapsed samples including OXPHOS pathways (Figure $3 \mathrm{~B} ; \mathrm{p}<0.01$ ). We also investigated the correlation of 18 mitochondrial data sets with drug resistance in the APEX trial including 169 relapsed MM samples [16]. The analyses of genome-wide gene expression data showed that MitoBio and OXPHOS signatures and another 6 pathways were increased in bortezomib-resistant MM samples compared to bortezomib-sensitive MM samples (Figure 3B; $\mathrm{p}<0.01$ ). However, we did not find a correlation between MitoBio signature and dexamethasone sensitivity (data not shown). These results suggest that MM patients with a high MitoBio signature and an activated OXPHOS pathway are resistant to bortezomib treatment.
A
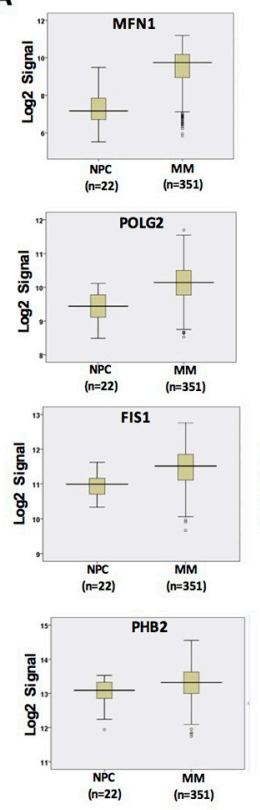

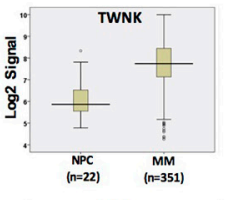

DRP1
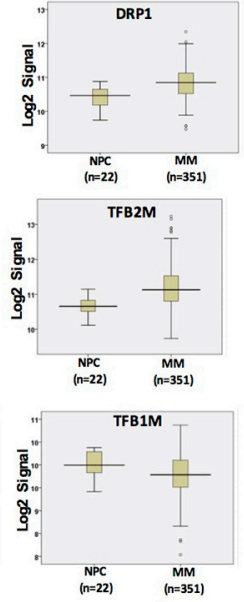
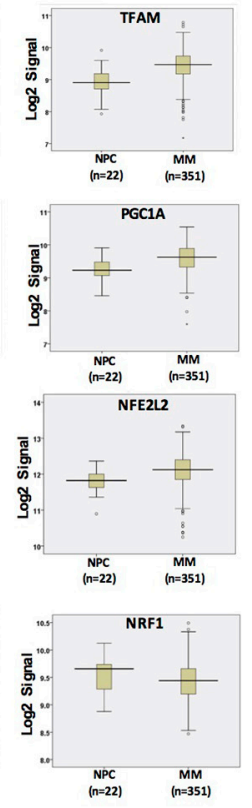
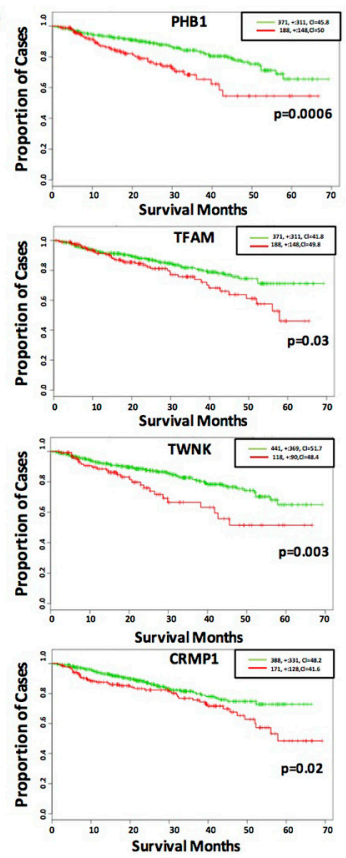
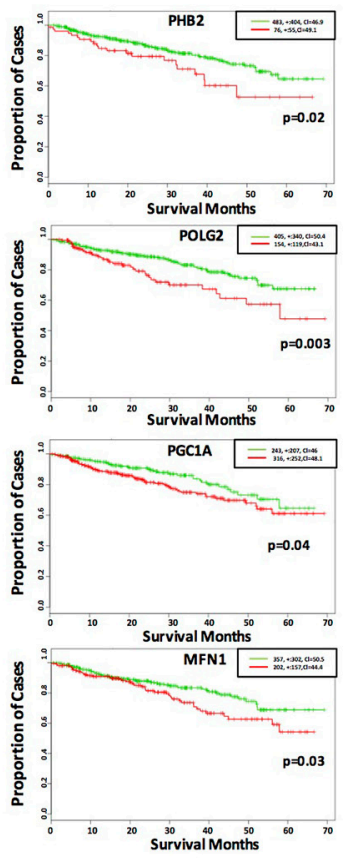

Figure 1: Dysregulated MitoBio genes in MM are associated with lower survival. (A) Gene expression profiles of 12 dysregulated MitoBio genes from 22 normal plasma cells (NPC) and 351 newly diagnosed MM samples in the total therapy 2 (TT2) clinical trial. (B) Kaplan-Meier survival analyses of 18 MitoBio genes were performed in 559 newly diagnosed MM samples. The correlation between GEP and survival was determined by the $\mathrm{p}$ value and hazard ratio (HR) at the best expression signal cut-off using SurvExpress website. 
A

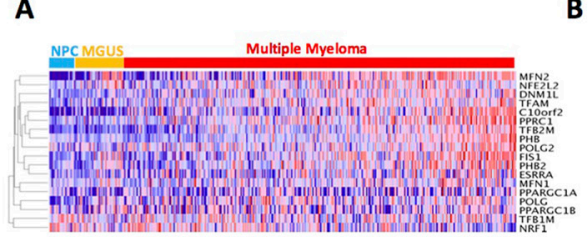

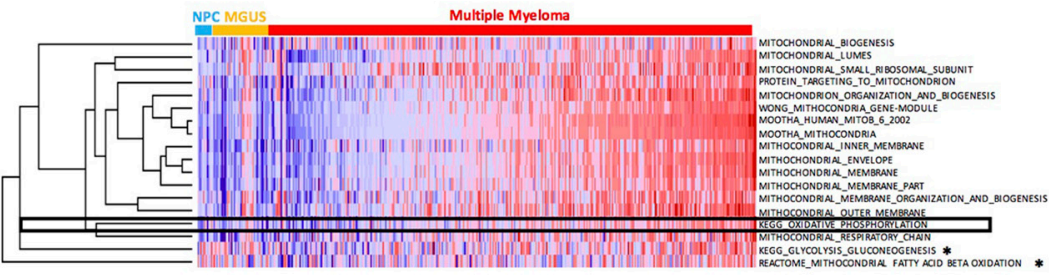

C
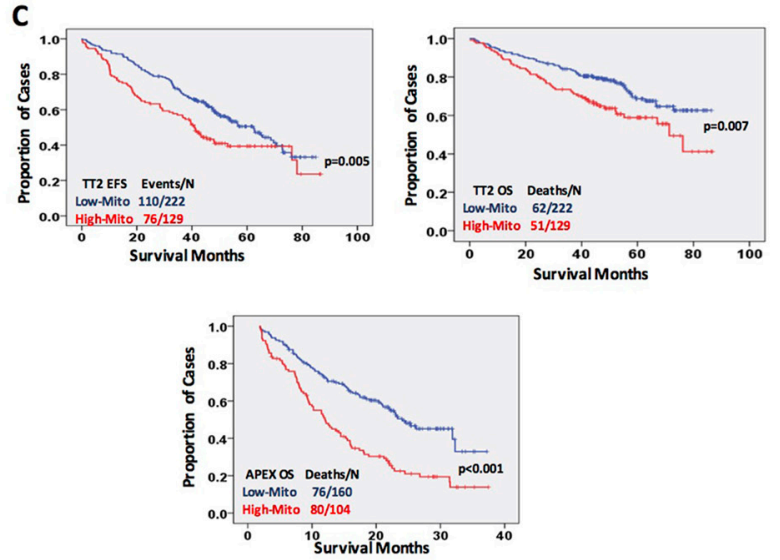

Figure 2: MitoBio signature is increased in newly diagnosed myeloma and links to a poor outcome. (A) Gene expression clustergram of 18 MitoBio genes in plasma cells from 22 healthy subjects (NPC), 44 MGUS and 351 newly diagnosed MM patients. (B) Single-sample gene set enrichment analysis performed in 22 NPC, 44 MGUS and 351 MM GEPs for MitoBio signature clustered with other 17 mitochondrial gene sets. MM samples were pre-ordered based on the ssGSEA scores of the gene set with all genes involved in the eighteen mitochondria-related pathways (Total-ssGSEA scores). Sixteen of the 18 sets are significantly upregulated in multiple myeloma $\left[\mathrm{p}<0.05 ;{ }^{*}\right.$ No statistic significance $\left.(\mathrm{p}>0.05)\right]$. (C) Kaplan-Meier analyses of event free survival (EFS) in TT2 cohort and overall survival (OS) in TT2 and APEX cohorts.

A

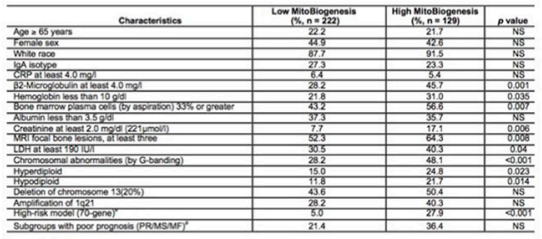

B

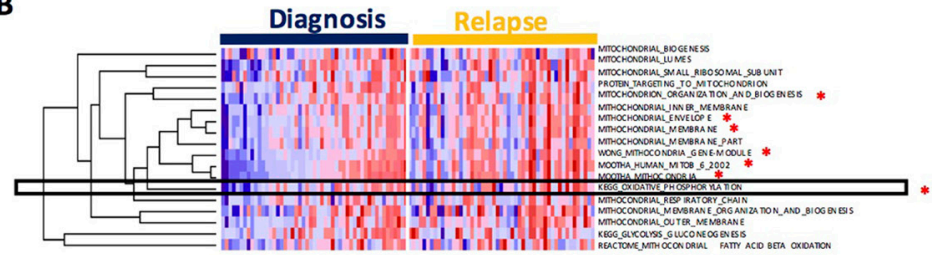

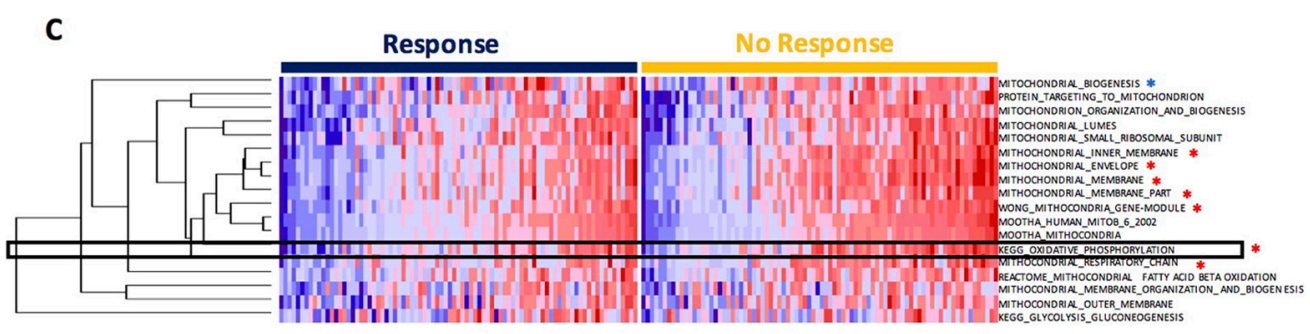

Figure 3: Drug resistant myeloma patients show higher expression of MitoBio gene sets. (A) Univeriate analysis was used to analyze the correlation of MitoBio score and clinical characteristics in TT2. (B) Single-sample gene set enrichment analysis performed in 51 paired GEPs collected at diagnosis and at relapse from $51 \mathrm{MM}$ patients enrolled in the TT2 cohort. Seven of the 18 sets show significantly upregulation $\left(^{*}\right)$. Samples were pre-ordered based on the Total-ssGSEA scores in diagnosis group. (C) Correlation of MitoBio genes with drug resistance in the APEX trial including 264 relapsed MM samples and 169 patients were treated with bortezomib. The MitoBio signature, oxidative phosphorylation and 6 other mitochondrial datasets $\left({ }^{*} \mathrm{p}<0.05\right)$ are increased in bortezomib-resistant MM samples (right) compared to bortezomib-sensitive MM samples (left). Samples were pre-ordered based on the Total-ssGSEA scores in each group. 


\section{Cellular iron content influences mitochondrial biogenesis and patient outcome in myeloma}

Disruption in iron homeostasis is often coupled with mitochondrial alteration. Because mitochondria are the major organelle to play a central role in iron-metabolism, we analyzed whether cellular iron influences MitoBio or vice versa. The expression of 18 MitoBio genes was compared in control $\mathrm{C} 2 \mathrm{C} 12$ cells and the same cells treated with the iron chelator desferoxamine (DFO) [17]. C2C12 is an immortalized mouse myoblast cell line widely used in biomedical research. Expression of the expression of iron exporter gene $F P N 1$ is downregulated in $\mathrm{C} 2 \mathrm{C} 12$ cells treated with DFO, while the iron importer gene $T F R C$ is upregulated (Figure 4A). Ten of 17 MitoBio genes (the gene Twinkle was not found on the Chip Annotation) including PHB1, PHB2, TFAM, TFB1M, TFB2M, PGC1B, $F I S 1, M F N 1, M F N 2$, and ESRRA were downregulated in DFO-treated $\mathrm{C} 2 \mathrm{C} 12$ cells; three genes $P O L G B, N R F 1$, and $N F E 2 L 2$ were upregulated in DFO-treated $\mathrm{C} 2 \mathrm{C} 12$ cells; and four genes $P O L G A, P G C 1 A, P P R C 1$, and $D R P 1$ were unchanged in DFO-treated C2C12 cells (Figure 4B). To determine whether increased MitoBio alters iron genes, the expression of FPN1 and TFRC was evaluated in the $\mathrm{C} 2 \mathrm{C} 12$ cells overexpressed with the PGC1A (PGC1A$\mathrm{OE})$. The expression of $T F R C$ is dramatically increased, while FPN1 was decreased in PGC1A-OE C2C12 cells (Figure 4C). Therefore, we conclude that both cellular iron and MitoBio are positively required for cellular physiological or pathological functions.

We have reported that most of iron-metabolism genes are dysregulated in MM cells [11]. Notably, FPN1 is significantly downregulated in MM cells compared to MGUS and NPCs and linked to a poor prognosis in MM. Based on results derived from $\mathrm{C} 2 \mathrm{C} 12$ cells, we wanted to determine whether dysregulated iron metabolism was related to an increased MitoBio in MM progression. By comparing high-FPN1 $(\mathrm{n}=88)$ with low-FPN1 $(\mathrm{n}=88)$ from 351 newly diagnosed MM samples, more than 500 genes were significantly differentially expressed between these groups and the top 100 genes are presented in the heatmap (Figure 5A). The GSEA showed the mitochondrial transcription pathway was ranked number 4 among the dysregulated pathways (Figure 5B; $\mathrm{p}<10^{-4}$ ) between high- and low-FPN1 MM samples. Importantly, the MitoBio genes, Twinkle, NFE2L2, POLG2, and PGC1A, were negatively correlated with the expression of FPN1 in primary MM cells (Figure $5 \mathrm{C}$ ). We further correlate the expression of FPN1 and TFRC with the mitochondrialscore in primary MM samples. As shown respectively in the Figure 6A, a high mitochondrial score was negatively correlated with the expression of FPN1 ( $\mathrm{p}<0.001)$, and positively correlated with the expression of TFRC (p < 0.001 ). To determine whether increased cellular iron and increased mitochondrial score have a synergistic effect in MM progression, Kaplan-Meier analyses were performed

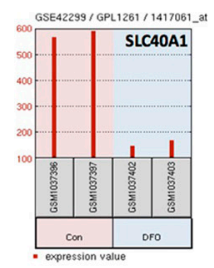

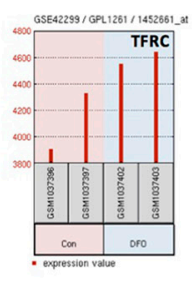

C

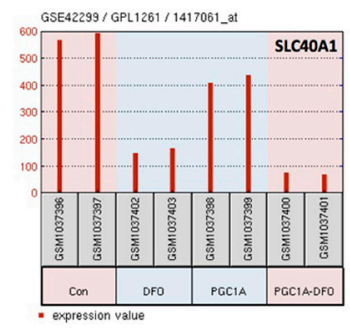

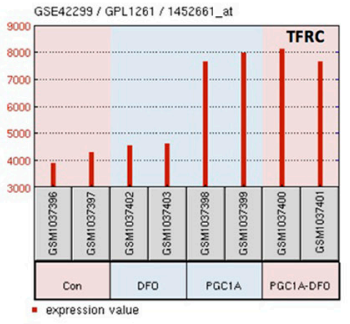

B
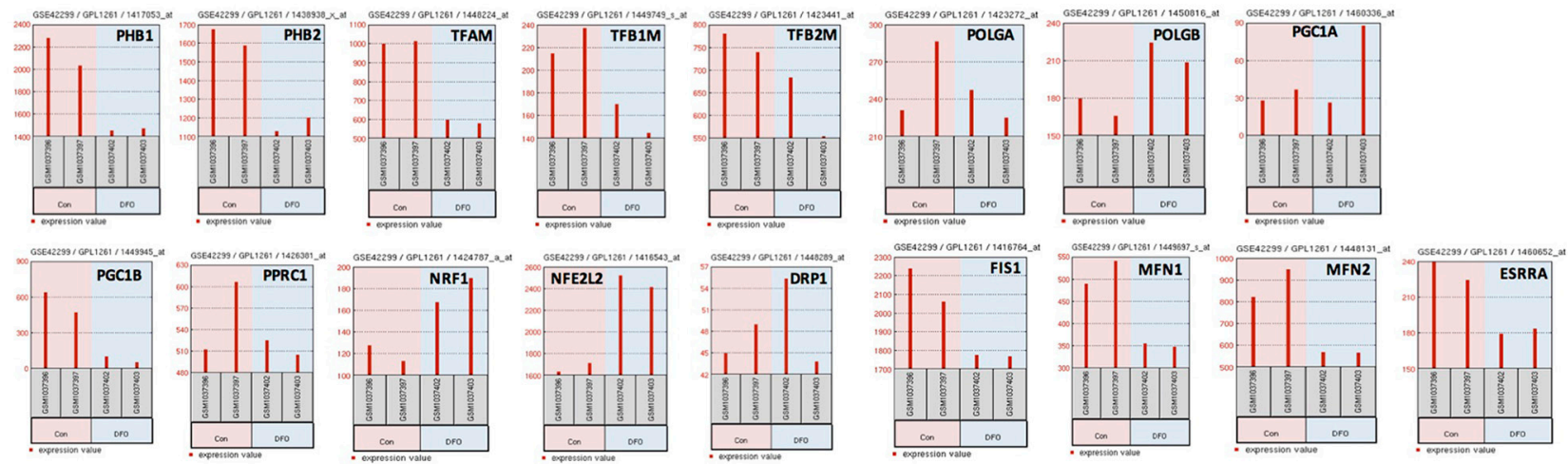

Figure 4: Iron influences mitochondrial biogenesis and patient outcome in myeloma. (A) The expression FPN1 (SLC40A, left panel) and TFRC (right panel) was analyzed in untreated and DFO-treated C2C12 cells determined by GEP. (B) FPN1 expression (SLC40A1, left panel) and TFRC (right panel) was analyzed in untreated and DFO-treated C2C12 cells overexpressing PGC1A. (C) Gene expression of 17 MitoBio genes in untreated and DFO-treated $\mathrm{C} 2 \mathrm{C} 12$ cells. 


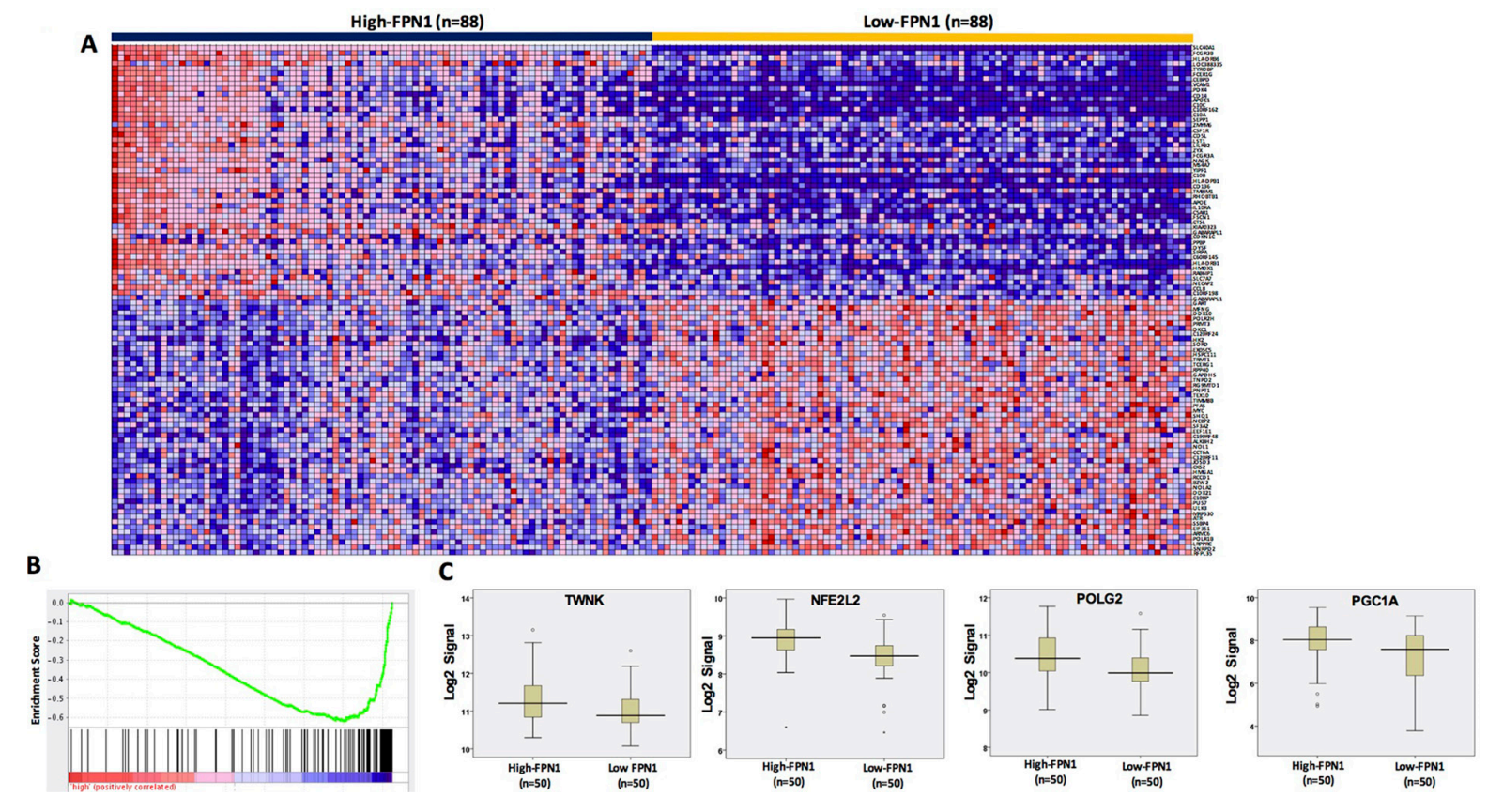

Figure 5: FPN1 dysregulation in MM alters MitoBio genes. (A) A gene expression clustergram of distinct genes in plasma cells between $88 \mathrm{MM}$ patients with high level or low FPN1 expression. (B) GSEA shows that the mitochondrial transcription pathway is significantly changed between high- and low-FPN1 expression. (C) Gene expression profiles of 4 MitoBio genes in 50 MM samples with high level or low of FPN1 expression $(\mathrm{p}<0.05)$.
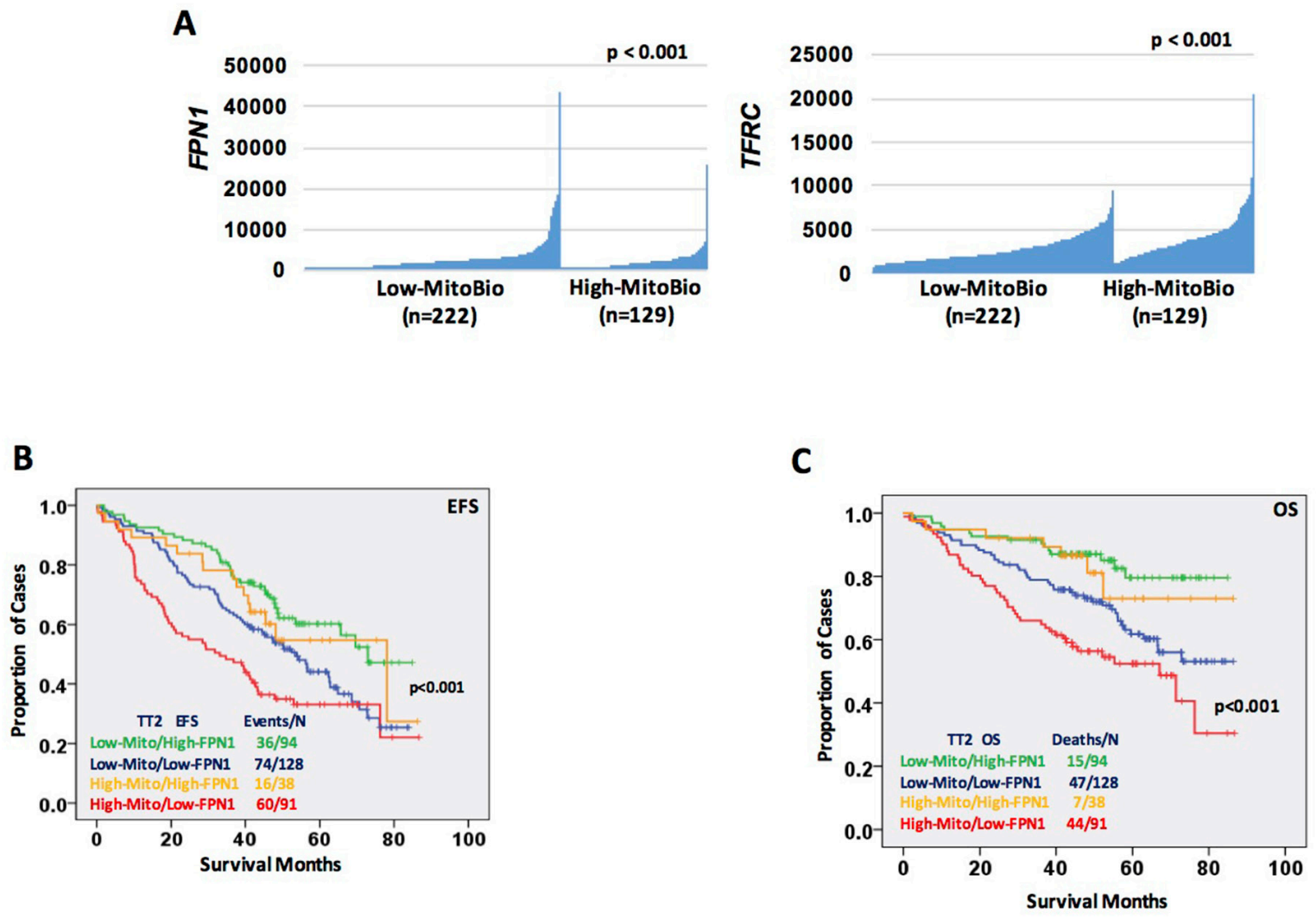

Figure 6: Increased cellular iron is positively correlated with dysregulated mitochondrial metabolism. (A) Correlation of FPN1 (SLC40A, D) and TFRC (E) expression within patients showing low mitochondrial-score ( $\mathrm{n}=222)$ or high mitochondrial-score $(\mathrm{n}=129)$. (B \& C) Kaplan-Meier analyses of free event survival (EFS, F) and overall survival (OS, G) of MM patients enrolled in TT2 cohort. Each line represents different combinations as described in the figure and color coded. 
between patients with different combinations of high- or low-FPN1 with high- or low-mitochondrial-score among the 351 newly diagnosed MM patients enrolled in the TT2 trial. The MM patients who have a high mitochondrialscore and a low-FPN1 signal show an inferior prognosis, whereas those who have a low mitochondrial-score and a high-FPN1 signal show the best prognosis in both EFS and OS (Figure 6B and $\mathrm{C}, \mathrm{p}<0.001$ ). Together, these data indicate that increased cellular iron is positively correlated with dysregulated mitochondrial metabolism, and synergizes for a poor outcome in MM.

\section{Pharmacological ascorbic acid induces myeloma cell death by targeting mitochondria}

AIF1 is a mitochondrial FAD-dependent oxidoreductase that plays a vital role in oxidative phosphorylation and redox metabolism in normal and cancer cells [18-22]. Because the OXPHOS pathway is significantly activated in both bortezomib-resistant and relapsed MM cells, and PAA, in the presence of iron, leads to the formation of ROS resulting in AIF1 cleavage and translocation from the mitochondria to the nucleus with consequent cell death, we hypothesized that the signaling pathway of MitoBio might be involved in PAA-induced MM death. PAA, > $1 \mathrm{mM}$, results in a 50- fold higher plasma levels than a physiological dose of ascorbate $(1 \mathrm{mg} /$ $\mathrm{kg}$ ) when administered intravenously [1]. In this study, we injected 1 x $10^{6}$ ARP1 MM cells intravenously in 15 NOD-Rag ${ }^{\text {null }}$ mice and started treating five mice with PAA $4 \mathrm{mg} / \mathrm{kg}$ intraperitoneal injections (five times/week for three weeks). Five mice were treated with PAA plus the iron chelator DFO, and another five mice treated with PBS as controls. DFO (100 mg/kg) was injected intraperitoneal once a day, (two days/week for three weeks). Tumor growth was significantly inhibited in the PAA-treated mice compared to those controls (Figure 7A \& B); while the combination of PAA with DFO did not show a significant difference in tumor growth compared to the control group $(\mathrm{p}>0.05)$. We also repeated this study by injecting $1 \times 10^{6}$ ARP1 MM cells subcutaneously in eight NOD$\mathrm{Rag}^{\text {null }}$ mice, four mice treated with PAA, and four mice as controls. Consistent with our previous results when MM cells were injected intravenously, we observed that tumor size was significantly smaller in the PAA-treated mice compared to those controls in these xenografted mice intraperitoneally injected (one mouse died from the control group) (Figure 7C). MM tumor cells were isolated from two mice in each group and microarrays were performed.

The ssGSEA analysis of 18 mitochondrial pathways indicated that PAA activates mitochondrial outer membrane and respiratory chain signaling pathways but inhibited small ribosomal subunit and oxidative phosphorylation signaling pathways (Figure 8A). We also performed western blots for AIF1 in ARP1 MM cells with or without PAA treatment and bortezomib was used as a negative control. As shown in the Figure 8B, nuclear AIF1 expression was significantly increased in PAA-treated MM cells compared to the control cells and the bortezomibtreated cells, further indicating that mitochondria were targeted by PAA. Since the oxidative phosphorylation pathway is significantly increased in both drug-resistant MM cells and relapsed MM samples, suggesting that PAA can overcome drug resistance to bortezomib by suppressing mitochondrial oxidative phosphorylation.

\section{DISCUSSION}

Here we report that the MitoBio signature is increased in MM cells compared to MGUS and normal plasma cells. Of the 18 MitoBio genes, eight are linked to an inferior overall survival including 559 newly diagnosed MM patients. A supervised hierarchical clustering further showed that a subset of MM patients has a high MitoBio signature compared to normal and MGUS samples. In addition to analyzing the 18 MitoBio genes, we also compared mitochondrial structure and functional signaling pathways including mitochondrial lumen, small ribosomal subunit, targeting to mitochondrion, organization and biogenesis, envelop, inner membrane, outer membrane, respiratory chain, oxidative phosphorylation, fatty acid $ß$-oxidation, and glycolysis gluconeogenesis between normal plasma cells and MM cells. As expected, the MM patients who have an increased MitoBio-related score show an early disease relapse and premature death. This is consistent with the report that melanoma patients with higher expression of MitoBio and tumor metabolism had worse overall survival [23]. Interestingly, all mitochondrial structure signaling pathways, and MitoBio and oxidative phosphorylation signatures are significantly upregulated in MM cells compared to normal plasma cells $(\mathrm{p}<0.001)$; while the signaling pathways of fatty acid $\beta$-oxidation and glycolysis gluconeogenesis do not have a difference between normal and tumor plasma cells. The supervised cluster in Figure $2 b$ shows clearly that both MitoBio and oxidative phosphorylation signatures are classified together with the mitochondrial structure signatures, but distinguished from fatty acid B-oxidation and glycolysis gluconeogenesis pathways. Although both oxidative phosphorylation and aerobic glycolysis play an important role in generating ATP for tumor cell growth, our data suggest that oxidative phosphorylation is the main pathway to provide energy for MM cell growth and proliferation.

To determine whether mitochondrial biogenesis is associated with disease relapse, ssGSEA analyses were performed on 51 paired MM samples collected at diagnosis and at relapse. Seven of 18 pathways were significantly upregulated in relapsed MM samples compared to those at diagnosis. To further determine whether this MitoBio is also related to drug resistance in 169 relapsed MM samples treated by bortezomib, eight of 18 pathways were 

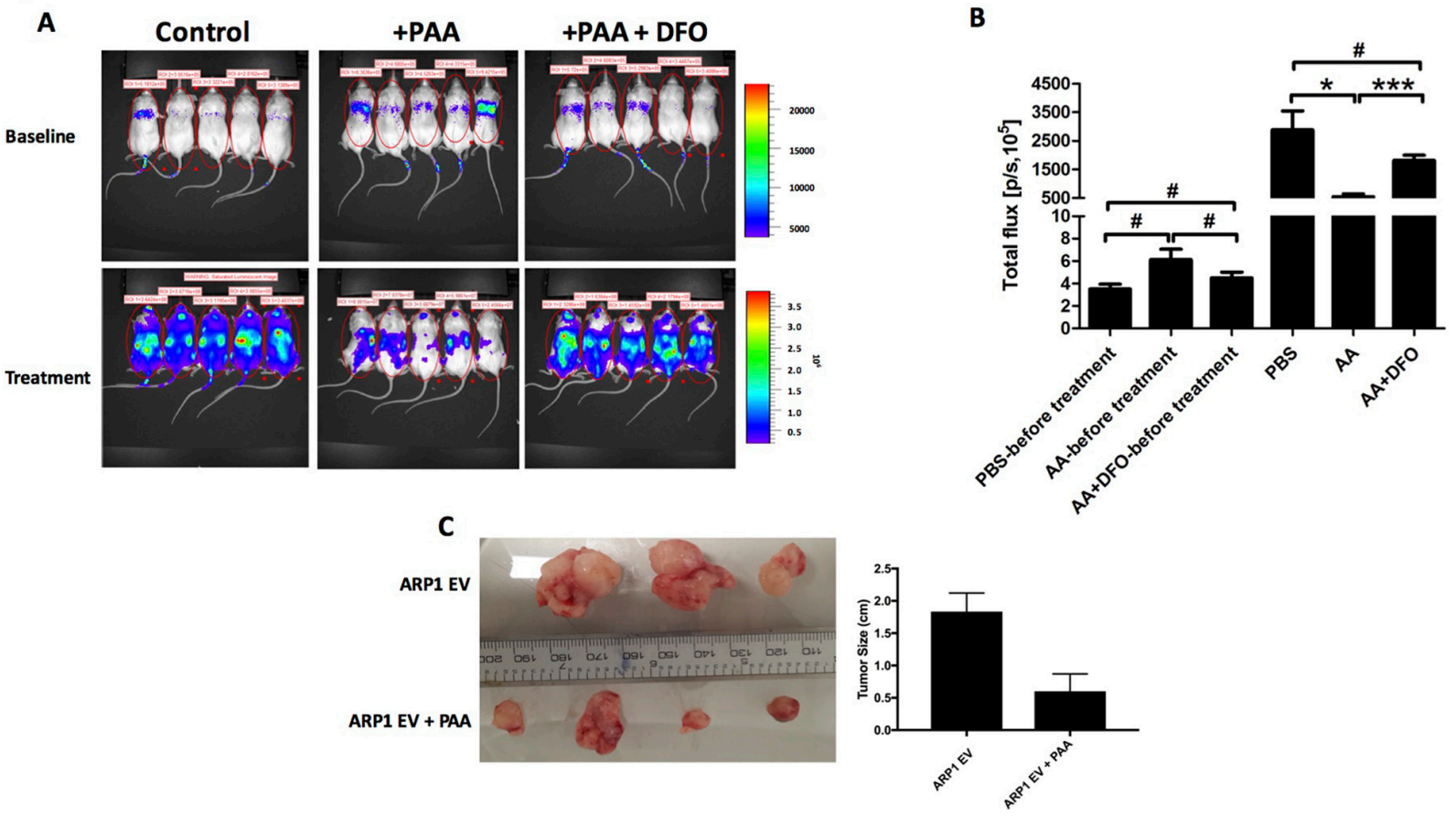

Figure 7: PAA prevents tumor growth in an iron-dependnet manner in MM. (A) Xenografted NOD.C $\gamma$-Rag1 mice (n=5/ group) were treated with PAA alone and in combination with DFO. After one-week injection of ARP1 cells, mice were treated with either PAA $(4 \mathrm{mg} / \mathrm{kg})$ injected intraperitoneal once a day, 5 days every week for 3 weeks. DFO $(100 \mathrm{mg} / \mathrm{kg})$ was injected intraperitoneal once a day, 2 days a week for 3 weeks. (B) Total flux of mice described in A indicates quantification of luciferase intensity (tumor burden) of mice pre- and post treatment at different time points. "No significance, ${ }^{*} \mathrm{p}<0.05,{ }^{* * *} \mathrm{p}<0.001$. (C) Another MM mouse model injected ARP1 intraperitoneally and treated with PAA was used and analyzed, tumors were dissected at week 3 after PAA treatment from each mouse. Tumor size was measured (right graph bar).

A

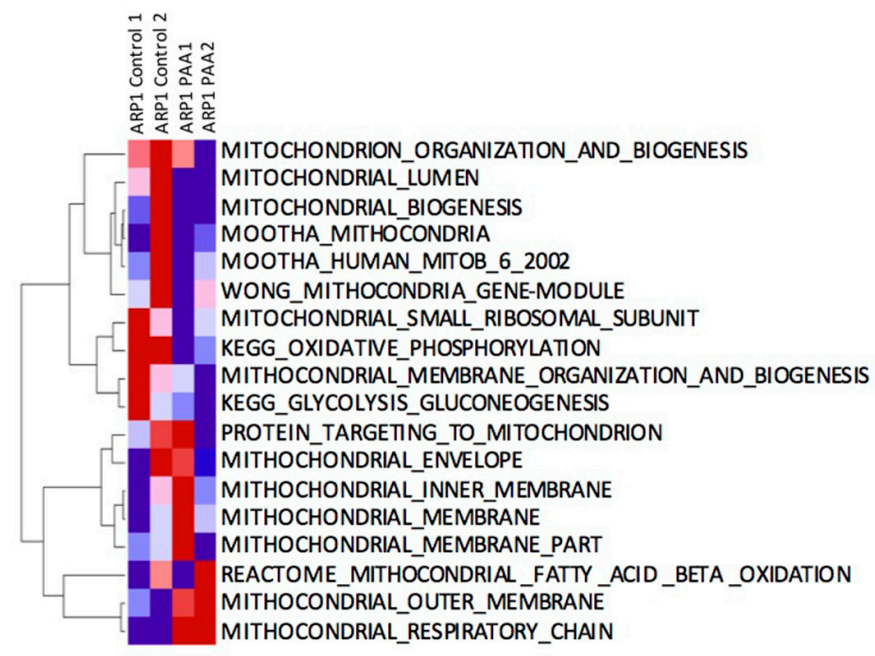

B

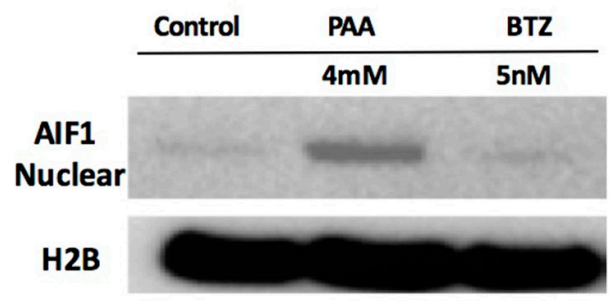

Figure 8: A. PAA inhibits mitochondrial oxidative phosphoryltion. (A) Total RNA was extracted from two mice from control (control 1,2) and PAA (PAA 1,2) groups for microarray. ssGSEA analysis of 18 mitochondrial gene datasets is shown. (B) ARP1 cells were incubated with or without PAA $(4 \mathrm{mM})$ or bortezomib (BTZ, 5nM). After $1 \mathrm{hr}$ PAA was washed away. After 6 hours, cellular fractionation was performed in each sample and nuclear AIF1 and H2B levels were analyzed by western blotting. 
increased in bortezomib-resistant MM samples, and five of them are commonly increased in both drug resistant and relapsed MM samples. We found that the direct MitoBio signature is only increased in bortezomib-resistant MM samples and not in the relapsed MM samples. However, the oxidative phosphorylation pathway is increased in both relapsed and drug resistant MM cells, supporting that the ATP provided by oxidative phosphorylation is not only important for MM disease development but also important for drug resistance and disease relapse in MM. We recently reported that PAA leads to the formation of ROS, resulting in AIF1 cleavage and translocation from the mitochondria to the nucleus, causing cell death. It is known that PAA induces cancer cell death and is dependent on depletion of ATP [24]. We compared these 18 mitochondrial signaling pathways before and after PAA treatment in MM cell line ARP1. We found that the OXPHOS signaling is one of the four pathways inhibited. Our data suggest that PAA inhibits OXPHOS signaling pathway and may cause a depletion of ATP resulting in MM cell death. PAA will be important to target drug resistant and relapsed MM in clinic.

The transcription co-activators, PGC1A and PGC1B, are the major proteins that enhance mitochondrial biogenesis, oxidative phosphorylation and the oxygen consumption rate $[25,26]$. c-Myc induces MitoBio through the activation of many MitoBio genes, especially dependent on PGC1B. PGC1A positively regulates a subset of OXPHOS genes in a time-dependent manner in a mouse skeletal muscle cell line [25]. Iron is essential for MitoBio and the iron importer gene transferrin receptor (TFRC) is a direct target of c-Myc. In our study, we found a positive correlation between cellular iron and the MitoBio signature. Depletion of cellular iron by DFO in $\mathrm{C} 2 \mathrm{C} 12$ cells caused the expression of most MitoBio genes to decrease, while overexpression of PGC1A in $\mathrm{C} 2 \mathrm{C} 12$ cells downregulated the expression of iron exporter gene FPN1 and upregulated the expression of TFRC. Consistently, MM samples with low FPN1 expression increased the expression of TWNK, NFE2L2, POLG1, and $P G C 1 A$, activated the mitochondrial transcription pathway. Importantly, MM patients with high MitoBio and low FPN1 had the worst clinical outcomes, indicating that iron is essential for MitoBio in MM cells for their biological functions, such as cell growth, proliferation and drug resistance. The possible reasons may be that both functional mitochondria and iron-sulfur cluster maintain nuclear genome stability [27], which are controlled by c-Myc in MM cells. We have recently shown that c-Myc also regulates the chromosomal instability (CIN) and drug resistant gene NEK2 in MM [28-30]. Our future work will explore whether c-Myc plays the key role in the dysregulated CIN in MM, MitoBio and iron metabolism, as well as how these pathways interact with each other.

Recent studies indicate that tumor cell growth and migration are fueled by enhanced mitochondrial biogenesis. Therefore, targeting MitoBio could be a strategy in cancer therapy. Our previous study showed that high-dosed ascorbic acid selectively kills MM cells including both primary MM cells and MM cell lines. In this study, we further determined that PAA inhibited MM cell growth dependent on cellular iron using an in vivo xenografted MM mouse model. This response process by PAA administration leads to mitochondrial swelling, the cristae disappeared and AIF1 cleavage and nuclear translocation was demonstrated by transmission electron microscopy (TEM) [1]. AIF1 is a mitochondrial FAD-dependent oxidoreductase, that plays a vital role in oxidative phosphorylation and redox metabolism in normal and cancer cells [18-22]. It is very likely that PAA generates high cellular ROS and inhibits mitochondrial AIF1 function as an oxidoreductase resulting in inhibition of oxidative phosphorylation and ATP depletion. In addition, AIF1 is an intermembrane space (IMS) component of the mitochondrial and characterized as a pro-apoptotic gene [31, 32]. The nuclear AIF1 or truncated AIF1 induced by PAA initiates chromatolysis and apoptosis-independent cell death [33]. Our data show that the proteasome inhibitor bortezomib, a widely-used drug in MM, has no effect on AIF1 expression, cleavage and localization; suggesting that PAA could be applied in MM patients, who are resistant to bortezomib. Targeting mitochondria proteins, such as CDDO and 10-TPP, etc., have been shown to enhance the chemotherapeutic effects in pre-clinical and clinical MM studies [2, 34, 35]. Future work will focus on the selection of those promising agents which have specific toxicity to MM cells but spare normal tissues.

\section{MATERIALS AND METHODS}

All methods were carried out in accordance with relevant institutional guidelines and regulations at the University of Iowa.

\section{Patient samples}

Gene expression profiles (GEP) of highly purified bone marrow plasma cells were analyzed from 559 newly diagnosed patients with MM including 351 from total therapy 2 (TT2) and 208 from TT3 cohorts. All consented patients' samples were collected according institutional approval. The protocol was approved by the institutional review board and the Food and Drug Administration as earlier described [36, 37]. The Gene Expression Omnibus database accession numbers described in this article were retrieved from GSE2658, GSE5900 and GSE9782. Kaplan-Meier analyses of event-free survival (EFS) and overall survival (OS) about patients in the TT2 and TT3 cohorts were performed using methods implemented in the SurvExpress website. Univariate analysis was used to test the associations between those mitochondrial biogenesis 
biomarkers and 19 clinical parameters in newly diagnosed myeloma patients.

\section{Gene set enrichment analysis (GSEA) and sSGSEA}

We performed GSEA and ssGSEA by using the applications available at the GenePattern platform of the Broad Institute of MIT and Harvard (https://genepattern. broadinstitute.org/gp/pages/index.jsf). For GESA, we estimated the signal-to-noise ratio and false discovery rates (FDR), from 1000 gene-set permutations. We tested the Gene ontology: Biological Process terms and KEGG pathways gene sets. For ssGSEA analysis, gene set activation scores for each sample were generated by online ssGSEA module based on the methodology described in Barbie et al., 2009 [38]. Raw enrichment scores were subjected to Hierarchiacal-Clustering analysis and heatmaps were generated by Hierachical-ClusteringImage module from Genepattern platform.

\section{Gene expression profiling (GEP)}

mRNA was extracted from ARP1 cell line before and after high-dosed ascorbic acid treatment using RNeasy kit (Qiagen, Valencia, CA, USA) accordingly with the manufacturer's instruction. GEP was performed on the Affymetrix Plus 2 chips on the $7 \mathrm{G}$ workstation and data was analyzed as previously reported $[13,39]$.

\section{Human myeloma cells in NOD.C $\gamma$-Rag1 mice}

The animal study was performed according to the guidelines of the Institutional Animal Care and local veterinary office and ethics committee of the University of Iowa, USA under approved protocol (IACUC 5081482). NOD.C $\gamma$-Rag1 mice 6-8 weeks old (Jackson Laboratory, Bar Harbor, ME, USA) were injected intravenously or intraperitoneally injection with ARP1 MM cells $(1 \times$ $10^{6}$ ) expressing luciferase. One-week after injection of ARP1 cells, mice were treated with either PAA (4 mg/ $\mathrm{kg}$ ) intraperitoneally injection once a day, 5 days every week for 3 weeks. DFO (100 $\mathrm{mg} / \mathrm{kg})$ was injected intraperitoneally once a day, 2 days a week for 3 weeks. The mice were euthanized when a humane endpoint was reached.

\section{Cell culture}

Human myeloma ARP1 cell line (kindly provided by Dr. John Shaughnessy, University of Arkansas for Medical Services, AR) was cultured in RPMI 1640 (Invitrogen, Carlsbad, CA, USA), supplemented with 10\% heat-inactivated FBS (Invitrogen), penicillin (100 IU/mL), and streptomycin $(100 \mu \mathrm{g} / \mathrm{mL})$ in a humidified incubator at $37{ }^{\circ} \mathrm{C}$ and $5 \% \mathrm{CO}_{2} / 95 \%$ air.

\section{Western blotting}

Cells were harvested and lysed with lysis buffer: $150 \mathrm{mM} \mathrm{NaCl}, 10 \mathrm{mM}$ EDTA, $10 \mathrm{mM}$ Tris, $\mathrm{pH}$ 7.4, $1 \%$ X-100 Triton. Cellular fractionation was performed according manufacturer's instruction (FractionPREP, BioVision). Cell lysates were subjected to SDS-PAGE, transferred onto a pure nitrocellulose membrane (BioRad) and blocked with 5\% fat-free milk. Primary antibodies for immunoblotting included: anti-AIF1 (1:1000, Cell Signaling) and and anti-H2B (1:1000, Cell Signaling) as loading control. Membranes were incubated with horseradish peroxidase (HRP)-conjugated anti-mouse secondary antibody $(1: 10,000$, Santa Cruz Biotechnology, cat\#: sc-2005) or anti-rabbit secondary antibody (1: 10,000, AnaSpec Inc., cat\#: AS-28177) for $1 \mathrm{~h}$ and chemiluminescence signals were detected by HRP substrate.

\section{Abbreviations}

MM: Multiple Myeloma; PAA: Pharmacological Ascorbic Acid; AIF1: Apoptosis-Inducing Factor 1; OXPHOS: oxidative phosphorylation; GEP: Gene Expression Profiles; MitoBio: Mitochondrial biogenesis; MGUS: Monoclonal gammopathy of undetermined significance; NPC: Normal Plasma Cells.

\section{Author contributions}

X.Z. analyzed the data, wrote and edited the paper, W.Y. and F.Z. generated the data and prepared figures, wrote and edited the manuscript; R.F.M., M.B., K.N., H.C., B.A.A., Y.J., I.F., G.T. discussed the results and edited the manuscript. All authors approved the final version of the manuscript.

\section{CONFLICTS OF INTEREST}

The authors declare no conflicts of interest.

\section{FUNDING}

This study was supported by NIH grant R01CA152105 (F.Z.), The Multiple Myeloma Research Foundation, the International Myeloma Foundation (F.Z.), the American Society of Hematology (ASH) Bridge (F.Z.), Holden Cancer University of Iowa Pilot Project and institutional start-up funds from the Department of Internal Medicine, Carver College of Medicine, University of Iowa (F.Z. \& G.T.).

\section{REFERENCES}

1. Xia J, Xu H, Zhang X, Allamargot C, Coleman KL, Nessler R, Frech I, Tricot G, Zhan F. Multiple Myeloma Tumor Cells are Selectively Killed by Pharmacologically-dosed 
Ascorbic Acid. EBioMedicine. 2017; 18:41-49. https://doi. org/10.1016/j.ebiom.2017.02.011.

2. Schibler J, Tomanek-Chalkley AM, Reedy JL, Zhan F, Spitz DR, Schultz MK, Goel A. Mitochondrial-Targeted DecylTriphenylphosphonium Enhances 2-Deoxy-D-Glucose Mediated Oxidative Stress and Clonogenic Killing of Multiple Myeloma Cells. PLoS One. 2016; 11:e0167323. https://doi.org/10.1371/journal.pone.0167323.

3. Dalton WS. Targeting the mitochondria: an exciting new approach to myeloma therapy. Commentary re: N. J. Bahlis et al., Feasibility and correlates of arsenic trioxide combined with ascorbic acid-mediated depletion of intracellular glutathione for the treatment of relapsed/refractory multiple myeloma. Clin. Cancer Res., 8: 3658-3668, 2002. Clin Cancer Res. 2002; 8:3643-45.

4. Chanan-Khan AA, Borrello I, Lee KP, Reece DE. Development of target-specific treatments in multiple myeloma. Br J Haematol. 2010; 151:3-15. https://doi. org/10.1111/j.1365-2141.2010.08262.x.

5. Zheng J. Energy metabolism of cancer: glycolysis versus oxidative phosphorylation (Review). Oncol Lett. 2012; 4:1151-57. Review.

6. Ploumi C, Daskalaki I, Tavernarakis N. Mitochondrial biogenesis and clearance: a balancing act. FEBS J. 2017; 284:183-95. https://doi.org/10.1111/febs.13820.

7. Palikaras K, Lionaki E, Tavernarakis N. Coordination of mitophagy and mitochondrial biogenesis during ageing in C. elegans. Nature. 2015; 521:525-28. https://doi. org/10.1038/nature14300.

8. Vyas S, Zaganjor E, Haigis MC. Mitochondria and Cancer. Cell. 2016; 166:555-66. https://doi.org/10.1016/j. cell.2016.07.002.

9. Hsieh AL, Dang CV. MYC, Metabolic Synthetic Lethality, and Cancer. Recent Results Cancer Res. 2016; 207:73-91. https://doi.org/10.1007/978-3-319-42118-6_4.

10. Paul BT, Manz DH, Torti FM, Torti SV. Mitochondria and Iron: current questions. Expert Rev Hematol. 2017; 10:6579. https://doi.org/10.1080/17474086.2016.1268047.

11. Gu Z, Wang H, Xia J, Yang Y, Jin Z, Xu H, Shi J, De Domenico I, Tricot G, Zhan F. Decreased ferroportin promotes myeloma cell growth and osteoclast differentiation. Cancer Res. 2015; 75:2211-21. https://doi. org/10.1158/0008-5472.CAN-14-3804.

12. Zhang G, Frederick DT, Wu L, Wei Z, Krepler C, Srinivasan S, Chae YC, Xu X, Choi H, Dimwamwa E, Ope O, Shannan B, Basu D, et al. Targeting mitochondrial biogenesis to overcome drug resistance to MAPK inhibitors. J Clin Invest. 2016; 126:1834-56. https://doi.org/10.1172/ JCI82661.

13. Zhan F, Huang Y, Colla S, Stewart JP, Hanamura I, Gupta S, Epstein J, Yaccoby S, Sawyer J, Burington B, Anaissie E, Hollmig K, Pineda-Roman M, et al. The molecular classification of multiple myeloma. Blood. 2006; 108:2020 28. https://doi.org/10.1182/blood-2005-11-013458.
14. Shaughnessy JD Jr, Zhan F, Burington BE, Huang Y, Colla S, Hanamura I, Stewart JP, Kordsmeier B, Randolph C, Williams DR, Xiao Y, Xu H, Epstein J, et al. A validated gene expression model of high-risk multiple myeloma is defined by deregulated expression of genes mapping to chromosome 1. Blood. 2007; 109:2276-84. https://doi. org/10.1182/blood-2006-07-038430.

15. Zhan F, Barlogie B, Arzoumanian V, Huang Y, Williams DR, Hollmig K, Pineda-Roman M, Tricot G, van Rhee F, Zangari M, Dhodapkar M, Shaughnessy JD Jr. Geneexpression signature of benign monoclonal gammopathy evident in multiple myeloma is linked to good prognosis. Blood. 2007; 109:1692-700. https://doi.org/10.1182/ blood-2006-07-037077.

16. Mulligan G, Mitsiades C, Bryant B, Zhan F, Chng WJ, Roels S, Koenig E, Fergus A, Huang Y, Richardson P, Trepicchio WL, Broyl A, Sonneveld P, et al. Gene expression profiling and correlation with outcome in clinical trials of the proteasome inhibitor bortezomib. Blood. 2007; 109:317788. https://doi.org/10.1182/blood-2006-09-044974.

17. Rensvold JW, Ong SE, Jeevananthan A, Carr SA, Mootha VK, Pagliarini DJ. Complementary RNA and protein profiling identifies iron as a key regulator of mitochondrial biogenesis. Cell Reports. 2013; 3:237-45. https://doi. org/10.1016/j.celrep.2012.11.029.

18. Sevrioukova IF. Apoptosis-inducing factor: structure, function, and redox regulation. Antioxid Redox Signal. 2011; 14:2545-79. https://doi.org/10.1089/ars.2010.3445.

19. Hangen E, Féraud O, Lachkar S, Mou H, Doti N, Fimia GM, Lam NV, Zhu C, Godin I, Muller K, Chatzi A, Nuebel E, Ciccosanti F, et al. Interaction between AIF and CHCHD4 Regulates Respiratory Chain Biogenesis. Mol Cell. 2015; 58:1001-14. https://doi.org/10.1016/j.molcel.2015.04.020.

20. Banci L, Bertini I, Cefaro C, Ciofi-Baffoni S, Gallo A, Martinelli M, Sideris DP, Katrakili N, Tokatlidis K. MIA40 is an oxidoreductase that catalyzes oxidative protein folding in mitochondria. Nat Struct Mol Biol. 2009; 16:198-206. https://doi.org/10.1038/nsmb.1553.

21. Pospisilik JA, Knauf C, Joza N, Benit P, Orthofer M, Cani PD, Ebersberger I, Nakashima T, Sarao R, Neely G, Esterbauer H, Kozlov A, Kahn CR, et al. Targeted deletion of AIF decreases mitochondrial oxidative phosphorylation and protects from obesity and diabetes. Cell. 2007; 131:476-91. https://doi.org/10.1016/j.cell.2007.08.047.

22. Vahsen N, Candé C, Brière JJ, Bénit P, Joza N, Larochette $\mathrm{N}$, Mastroberardino PG, Pequignot MO, Casares N, Lazar V, Feraud O, Debili N, Wissing S, et al. AIF deficiency compromises oxidative phosphorylation. EMBO J. 2004; 23:4679-89. https://doi.org/10.1038/sj.emboj.7600461.

23. Zhang H, Hilton MJ, Anolik JH, Welle SL, Zhao C, Yao Z, Li X, Wang Z, Boyce BF, Xing L. NOTCH inhibits osteoblast formation in inflammatory arthritis via noncanonical NF-кB. J Clin Invest. 2014; 124:3200-14. https://doi.org/10.1172/JCI68901. 
24. Chen P, Yu J, Chalmers B, Drisko J, Yang J, Li B, Chen Q. Pharmacological ascorbate induces cytotoxicity in prostate cancer cells through ATP depletion and induction of autophagy. Anticancer Drugs. 2012; 23:437-44. https:// doi.org/10.1097/CAD.0b013e32834fd01f.

25. Mootha VK, Lindgren CM, Eriksson KF, Subramanian A, Sihag S, Lehar J, Puigserver P, Carlsson E, Ridderstråle M, Laurila E, Houstis N, Daly MJ, Patterson N, et al. PGC-1alpha-responsive genes involved in oxidative phosphorylation are coordinately downregulated in human diabetes. Nat Genet. 2003; 34:267-73. https://doi. org/10.1038/ng1180.

26. LeBleu VS, O'Connell JT, Gonzalez Herrera KN, Wikman H, Pantel K, Haigis MC, de Carvalho FM, Damascena A, Domingos Chinen LT, Rocha RM, Asara JM, Kalluri R. PGC-1alpha mediates mitochondrial biogenesis and oxidative phosphorylation in cancer cells to promote metastasis. Nat Cell Biol. 2014; 16:992-1003.

27. Veatch JR, McMurray MA, Nelson ZW, Gottschling DE. Mitochondrial dysfunction leads to nuclear genome instability via an iron-sulfur cluster defect. Cell. 2009; 137:1247-58. https://doi.org/10.1016/j.cell.2009.04.014 PMID:19563757.

28. Zhou W, Yang Y, Xia J, Wang H, Salama ME, Xiong W, Xu H, Shetty S, Chen T, Zeng Z, Shi L, Zangari M, Miles $\mathrm{R}$, et al. NEK2 induces drug resistance mainly through activation of efflux drug pumps and is associated with poor prognosis in myeloma and other cancers. Cancer Cell. 2013; 23:48-62. https://doi.org/10.1016/j.ccr.2012.12.001 PMID:23328480.

29. Zhou W, Yang Y, Gu Z, Wang H, Xia J, Wu X, Zhan X, Levasseur D, Zhou Y, Janz S, Tricot G, Shi J, Zhan F. ALDH1 activity identifies tumor-initiating cells and links to chromosomal instability signatures in multiple myeloma. Leukemia. 2014; 28:1155-58. https://doi.org/10.1038/ leu.2013.383 PMID:24365790.

30. Gu Z, Xia J, Xu H, Frech I, Tricot G, Zhan F. NEK2 Promotes Aerobic Glycolysis in Multiple Myeloma Through Regulating Splicing of Pyruvate Kinase. J Hematol Oncol. 2017; 10:17. https://doi.org/10.1186/s13045-017-0392-4 PMID:28086949.

31. Susin SA, Lorenzo HK, Zamzami N, Marzo I, Snow BE, Brothers GM, Mangion J, Jacotot E, Costantini P, Loeffler M, Larochette N, Goodlett DR, Aebersold R, et al. Molecular characterization of mitochondrial apoptosisinducing factor. Nature. 1999; 397:441-46. https://doi. org/10.1038/17135 PMID:9989411.

32. Joza N, Susin SA, Daugas E, Stanford WL, Cho SK, Li CY, Sasaki T, Elia AJ, Cheng HY, Ravagnan L, Ferri
KF, Zamzami N, Wakeham A, et al. Essential role of the mitochondrial apoptosis-inducing factor in programmed cell death. Nature. 2001; 410:549-54. https://doi. org/10.1038/35069004 PMID:11279485.

33. Artus C, Boujrad H, Bouharrour A, Brunelle MN, Hoos S, Yuste VJ, Lenormand P, Rousselle JC, Namane A, England P, Lorenzo HK, Susin SA. AIF promotes chromatinolysis and caspase-independent programmed necrosis by interacting with histone H2AX. EMBO J. 2010; 29:1585-99. https://doi.org/10.1038/emboj.2010.43 PMID:20360685.

34. Chauhan D, Li G, Podar K, Hideshima T, Shringarpure R, Catley L, Mitsiades C, Munshi N, Tai YT, Suh N, Gribble GW, Honda T, Schlossman R, et al. The bortezomib/ proteasome inhibitor PS-341 and triterpenoid CDDO-Im induce synergistic anti-multiple myeloma (MM) activity and overcome bortezomib resistance. Blood. 2004; 103:3158-66. https://doi.org/10.1182/blood-2003-08-2873 PMID:15070698.

35. Dvorakova K, Waltmire CN, Payne CM, Tome ME, Briehl MM, Dorr RT. Induction of mitochondrial changes in myeloma cells by imexon. Blood. 2001; 97:3544-51. https:// doi.org/10.1182/blood.V97.11.3544 PMID:11369649.

36. Barlogie B, Tricot G, Anaissie E, Shaughnessy J, Rasmussen E, van Rhee F, Fassas A, Zangari M, Hollmig K, Pineda-Roman M, Lee C, Talamo G, Thertulien R, et al. Thalidomide and hematopoietic-cell transplantation for multiple myeloma. N Engl J Med. 2006; 354:1021-30. https://doi.org/10.1056/NEJMoa053583 PMID:16525139.

37. Barlogie B, Anaissie E, van Rhee F, Pineda-Roman M, Zangari M, Shaughnessy J, Epstein J, Crowley J. The Arkansas approach to therapy of patients with multiple myeloma. Best Pract Res Clin Haematol. 2007; 20:761-81. https://doi.org/10.1016/j.beha.2007.09.005 PMID:18070718.

38. Barbie DA, Tamayo P, Boehm JS, Kim SY, Moody SE, Dunn IF, Schinzel AC, Sandy P, Meylan E, Scholl C, Fröhling S, Chan EM, Sos ML, et al. Systematic RNA interference reveals that oncogenic KRAS-driven cancers require TBK1. Nature. 2009; 462:108-12. https://doi. org/10.1038/nature08460 PMID:19847166.

39. Zhan F, Hardin J, Kordsmeier B, Bumm K, Zheng M, Tian E, Sanderson R, Yang Y, Wilson C, Zangari M, Anaissie E, Morris C, Muwalla F, et al. Global gene expression profiling of multiple myeloma, monoclonal gammopathy of undetermined significance, and normal bone marrow plasma cells. Blood. 2002; 99:1745-57. https://doi. org/10.1182/blood.V99.5.1745 PMID:11861292. 